\title{
Desafios dos atendimentos não presenciais de Fisioterapia na pandemia da COVID-19
}

\author{
Challenges of non-face-to-face physiotherapy care in the pandemic of COVID-19
}

Retos de la atención fisioterapéutica no presencial en la pandemia de COVID-19

\section{Resumo}

Introdução: O mundo está atualmente passando por uma pandemia causada por um novo coronavírus (SARS-CoV-2), que causa a doença Coronavírus disease 2019(COVID-19). Devido sua alta transmissibilidade várias atividades econômicas e de saúde foram suspensas. A ciência fisioterapêutica não ficou imune. O Conselho Federal de Fisioterapia e Terapia Ocupacional autorizou o atendimento não presencial nas modalidades teleconsulta, teleconsultoria e telemonitoramento. O objetivo deste trabalho é buscar na literatura os desafios dos atendimentos não presenciais de fisioterapia no contexto da pandemia da COVID-19. Metodologia: Trata-se de uma revisão de literatura, a qual a extração das informações foi realizada através de um levantamento bibliográfico e análise dos artigos relevantes sobre a temática. Resultado: os estudos utilizados na construção deste artigo afirmam em sua totalidade resultados positivos na aplicação de atendimentos não presenciais por meio de teleconsultas. Conclusão: A telessaúde tem sido uma importante alternativa para os profissionais da saúde garantirem o acompanhamento de seus pacientes em tempo real neste período de pandemia da COVID-19. Todavia, esta ferramenta detém aspectos negativos como a limitação de acesso à tecnologia e Internet de qualidade, dentre outros aspectos. Assim o atendimento fisioterapêutico de forma remota, se depara com desafios significativos que devem ser estudados a fundo e o uso da telessaúde pode ser mais proficiente em um futuro bem próximo.

Palavras-chave: Fisioterapia; COVID-19; Teleconsulta.

\begin{abstract}
Introduction: The world is currently experiencing a pandemic caused by a new coronavirus (SARS-CoV-2), which causes coronavirus disease disease 2019 (COVID-19). Due to its high transmissibility several economic and health activities were suspended. The physiotherapeutic science was not immune. The Federal Council of Physiotherapy and Occupational Therapy authorized non-face-to-face care in the modalities of teleconsultation, teleconsulting and telemonitoring. The aim of this work is to seek in the literature the challenges of non-face-to-face physiotherapy care in the context of the COVID-19 pandemic. Methodology: This is a literature review, which was obtained through a bibliographic survey and analysis of the relevant articles on the subject. Result: the studies used in the construction of this article state in their entirety positive results in the application of non-face-to-face care through teleconsultations. Conclusion: Telessaúde has been an important alternative for health professionals to ensure the follow-up of their patients in real time in this period of pandemic COVID-19. However, this tool has negative aspects such as the limitation of access to quality technology and Internet, among other aspects. Thus, physical therapy care in a remote way is faced with significant challenges that must be studied in depth and the use of low-health may be more proficient in the very near future.
\end{abstract}

Keywords: Physiotherapy; COVID-19; Teleconsultation. 


\begin{abstract}
Resumen
Introducción: El mundo vive actualmente una pandemia causada por un nuevo coronavirus (SARS-CoV-2), causante de la enfermedad por coronavirus 2019 (COVID-19). Debido a su alta transmisibilidad se suspendieron varias actividades económicas y sanitarias. La ciencia fisioterapéutica no era inmune. El Consejo Federal de Fisioterapia y Terapia Ocupacional autorizó la atención no presencial en las modalidades de teleconsulta, teleconsulta y telemonitorización. El objetivo de este trabajo es buscar en la literatura los retos de la atención fisioterapéutica no presencial en el contexto de la pandemia de COVID-19. Metodología: Se trata de una revisión bibliográfica, que se obtuvo a través de un estudio bibliográfico y análisis de los artículos relevantes sobre el tema. Resultado: los estudios utilizados en la construcción de este artículo declaran en su totalidad resultados positivos en la aplicación de la atención no presencial a través de teleconsultas. Conclusión: Telessaúde ha sido una alternativa importante para que los profesionales de la salud aseguren el seguimiento de sus pacientes en tiempo real en este periodo de pandemia de COVID-19. Sin embargo, esta herramienta tiene aspectos negativos como la limitación del acceso a tecnología de calidad e Internet, entre otros aspectos. Por lo tanto, la atención de la fisioterapia de manera remota se enfrenta a desafíos significativos que deben ser estudiados en profundidad y el uso de la baja salud puede ser más competente en un futuro muy cercano.
\end{abstract}

Palabras clave: Fisioterapia; COVID-19; Teleconsulta.

\title{
1. Introdução
}

O mundo está atualmente passando por uma pandemia causada por um novo coronavírus (SARS-CoV-2), que causa a doença Coronavírus disease 2019(COVID-19). Após os primeiros casos relatados na China em dezembro de 2019, o novo coronavírus se espalhou rapidamente, causando danos incalculáveis à qualidade de vida (Sharid et al., 2020).

Em 29 de março de 2020 mais de 700000 casos já foram confirmados em todo o mundo. Estes relatos indicam a rapidez de espalhamento da nova doença por coronavírus (COVID-19) causada pela infecção pelo vírus SARS-COV-2, categorizada como uma pandemia em 11 de março de 2020 pela Organização Mundial de Saúde. No Brasil, os primeiros casos foram confirmados no mês de fevereiro, e diversas ações foram implementadas a fim de conter e de mitigar o avanço da doença. Em 3 de fevereiro de 2020, o país declarou Emergência de Saúde Pública de Importância Nacional (ESPIN),13 antes mesmo da confirmação do primeiro caso (OMS, 2020; Brasil, 2020).

SARS-CoV-2 é altamente transmissível por gotículas e contato, principalmente em locais fechados e ambientes hospitalares. Um indivíduo com infecção pelo novo coronavírus transmite para outras duas ou três pessoas, dependendo das condições ambientais. Locais fechados com pouca ventilação e baixa luminosidade facilitam a transmissão do vírus. As pessoas com COVID-19 podem apresentar tosse, dificuldade para respirar, dores de garganta, febre e outras manifestações clínicas. Há ainda os portadores assintomáticos, os quais possuem importância epidemiológica, dado que são potenciais transmissores (Medeiros, 2020; Surveillances, 2020). Zhang (2020) e Lloyd-sherlock et al. (2020) em seus estudos apontam que o risco de morte é maior em idosos em especial aqueles com comorbidades.

Durante essa pandemia, o mundo e o Brasil adotaram medidas de isolamento e distanciamento social, interrupção de aulas e trabalhos presenciais, gerando além do sofrimento com o noticiário de mortes e hospitalização, danos emocionais e financeiros que afetaram diferentemente cada grupo geracional. A ciência fisioterapêutica não ficou imune. Sendo uma das áreas que mais carecem do contato físico, automaticamente fora uma das mais afetadas com a pandemia que tem como principal característica o isolamento social (Zhang, 2020; Soares E Fiorino, 2020).

O Conselho Federal de Fisioterapia e Terapia Ocupacional (COFFITO), editou a resolução n ${ }^{\circ} 516$, de 20 de março de 2020, na qual estabelece permissão para atendimento não presencial nas modalidades teleconsulta, teleconsultoria e telemonitoramento. De acordo com o parágrafo 1, a teleconsulta consiste na consulta clínica registrada e realizada pelo fisioterapeuta e terapeuta ocupacional à distância. O parágrafo 2 diz que o telemonitoramento consiste no acompanhamento à distância, de paciente atendido previamente no presencial. O parágrafo 3 trata da teleconsultoria, que consiste na comunicação registrada e realizada entre profissionais e gestores, com a finalidade de esclarecer dúvidas sobre procedimentos clínicos, ações de saúde e questões relativas ao processo de trabalho em meio a pandemia (COFFITO, 2020). 
Mediante os fatos apresentados, surge o seguinte questionamento: quais os desafios encontrados nos trabalhos não presenciais da fisioterapia na pandemia da Covid-19?

Portanto, o objetivo deste trabalho é buscar na literatura os desafios dos atendimentos não presenciais de fisioterapia no contexto da pandemia da COVID-19.

\section{Metodologia}

Trata-se de uma revisão integrativa da literatura que de acordo com Mendes et al., 2008 é um tipo de estudo que sintetiza resultados relevantes sobre determinado tema. A extração das informações foi realizada através de um levantamento bibliográfico e análise dos artigos relevantes sobre a temática. As buscas foram realizadas nas bases de dados Pubmed e SciELO, utilizando os descritores: Fisioterapia, Teleconsulta e COVID-19. Os critérios de inclusão foram: artigos com publicação no ano 2020 nos idiomas Inglês, Português e Espanhol.

A análise dos artigos se deu primariamente através do título e aqueles que não tinham nenhuma relação com o tema do presente artigo, foram excluídos da pesquisa. A seleção dos artigos se deu pela leitura completa, com exclusão daqueles que não preenchiam os critérios de inclusão. Ao todo 6 artigos foram incluídos no presente estudo.

A estratégia de buscas se baseou nos descritores "Fisioterapia and teleconsulta and COVID-19." No Pubmed, utilizando os descritores: teleconsulta and fisioterapia and COVID-19, foram encontrados 5 artigos, dos quais apenas 2 foram selecionados. Na primeira busca no SciELO, utilizando os descritores: fisioterapia and COVID-19, foram encontrados 10 artigos, dos quais apenas 1 foi selecionado. Na segunda busca no SciELO, utilizando os descritores: fisioterapia and teleconsulta, foram encontrados 1, o qual foi selecionado. Por fim, na busca avançada no Google Acadêmico, utilizando os descritores: fisioterapia and teleconsulta and COVID-19, foram encontrados 77 artigos, dos quais apenas 2 foram selecionados.

\section{Resultados}

A seguir apresenta-se o Quadro 1 que contém os seis artigos encontrados, os seus títulos, autores, tipos de estudo e os seus resultados.

Quadro 1: Artigos selecionados após a estratégia de busca submetida. 2020.

\begin{tabular}{|c|c|c|c|}
\hline TÍTULO & AUTOR & $\begin{array}{l}\text { TIPO DE } \\
\text { ESTUDO }\end{array}$ & RESULTADO \\
\hline $\begin{array}{lr}\text { Musculoskeletal } & \text { Physical } \\
\text { Therapy Duringthe } & \text { COVID- } \\
19 \quad \text { Pandemic: r Is } \\
\text { Telerehabilitation } \\
\text { Answer? }\end{array}$ & TUROLLA et al.2020 & Revisão literária & $\begin{array}{l}\text { A telerreabilitação se revela um modelo } \\
\text { promissor e oportuno de atendimento a ser } \\
\text { adotado alternativamente, ou em combinação } \\
\text { com o atendimento presencial usual para } \\
\text { pacientes com disfunção MSK durante a } \\
\text { pandemia de COVID-19 }\end{array}$ \\
\hline $\begin{array}{l}\text { Estimating the impactof } \\
\text { COVID-19 pandemic on } \\
\text { services provided by Italian } \\
\text { Neuromuscular Centers: na } \\
\text { Italian Association of Mylogy } \\
\text { survey of the acutephase }\end{array}$ & MAURI et al, 2020 & $\begin{array}{l}\text { Estudo transversal } \\
\text { baseado em uma } \\
\text { pesquisa } \\
\text { multicêntrica }\end{array}$ & $\begin{array}{l}\text { Apesar dos esforços para fornecer consultas de } \\
\text { telemedicina aos pacientes, esta opção poderia ser } \\
\text { promovida e melhorada ainda mais com uma } \\
\text { estreita colaboração entre os diferentes centros } \\
\text { neuromusculares e prestadores de serviços, bem } \\
\text { como a implementação adicional de plataformas } \\
\text { de telessaúde. }\end{array}$ \\
\hline $\begin{array}{lr}\text { Digital Physical } & \text { Therapy in } \\
\text { Gynecologic } & \text { Oncology } \\
\text { during the } & \text { COVID-19 } \\
\text { Pandemic } & \end{array}$ & $\begin{array}{l}\text { CARVALHO et al. } \\
2020\end{array}$ & Revisão literária & $\begin{array}{l}\text { A fisioterapia digital por meio de tele-consulta e } \\
\text { tele-monitoramento é um ativo promissor para } \\
\text { manter o cuidado às pacientes com câncer } \\
\text { ginecológico, evitando a descontinuação do } \\
\text { acompanhamento neste momento, minimizando o } \\
\text { comprometimento dos resultados, reduzindo o } \\
\text { trânsito para hospitais / ambulatórios instalações, } \\
\text { risco de contaminação e favorecimento da } \\
\text { funcionalidade dessas mulheres }\end{array}$ \\
\hline
\end{tabular}




\begin{tabular}{|l|l|l|l|}
\hline $\begin{array}{l}\text { Direito e fisioterapia: } \\
\text { reflexões acerca dos } \\
\text { atendimentos fisioterápicos } \\
\text { não presenciais à luz do } \\
\text { direito à saúde do paciente em } \\
\text { tempos pandêmicos }\end{array}$ & $\begin{array}{l}\text { SOARS } \\
\text { FIORINDO, 2020 }\end{array}$ & $\begin{array}{l}\text { Revisão } \\
\text { bibliográfica }\end{array}$ & $\begin{array}{l}\text { O sistema de atendimento não presencial deve ser } \\
\text { visto não somente como uma medida adequada a } \\
\text { ocasiões excepcionais, mas como um modelo } \\
\text { efetivo a ser utilizado na rotina do fisioterapeuta, } \\
\text { ao passo que se apresenta como mais um meio } \\
\text { colocado à sua disposição para garantir ao } \\
\text { paciente uma melhor qualidade de vida, em todas } \\
\text { as suas vertentes }\end{array}$ \\
\hline $\begin{array}{l}\text { Physiotherapy services in the } \\
\text { face of a pandemic. }\end{array}$ & MINGHELLI, 2020 & Estudo de campo & $\begin{array}{l}\text { A maioria dos fisioterapeutas interromperam as } \\
\text { suas práticas presenciais em virtude da pandemia } \\
\text { COVID-19, no entanto, apesar de não estarem a } \\
\text { acompanhar os seus pacientes pessoalmente, a } \\
\text { maioria deles elaborou ferramentas para } \\
\text { monitorar seus pacientes à distância. }\end{array}$ \\
\hline $\begin{array}{l}\text { Teleconsultation in a } \\
\text { pandemic dueto Coronavirus: } \\
\text { challenges for telemedicine in } \\
\text { the post - COVID-19 era. }\end{array}$ & MÁRQUEZ,2020 & $\begin{array}{l}\text { Estudo } \\
\text { observacional }\end{array}$ & $\begin{array}{l}\text { A teleconsulta, proporciona aos pacientes um } \\
\text { atendimento mais conveniente e econômico. A } \\
\text { telemedicina é uma grande oportunidade para } \\
\text { melhorar os sistemas de apoio à saúde }\end{array}$ \\
\hline
\end{tabular}

Fonte: Dados dos autores (2020).

Percebe-se que no Quadro 1 os autores trazem resultados positivos acerca do uso da teleconsulta na pandemia da COVID-19, todavia o autor Mauri et al, 2020 sugere melhorias nesse tipo de atendimento.

\section{Discussão}

\subsection{Possibilidades e potencialidades do uso de teleatendimento em períodos de pandemia.}

Durante a pandemia do novo coronavírus os governos instituíram medidas protetivas de isolamento social objetivando desacelerar o contágio e consequente aumento de pessoas hospitalizadas temendo que as estruturas hospitalares não fossem suficientes para atender à população e causasse um colapso na saúde. Além dos pacientes infectados por Covid, não se pode negligenciar aqueles que se encontram em tratamento de saúde devido outras patologias. A utilização de consultas a distância foi uma das alternativas encontradas para continuar atendendo àqueles que necessitavam de tratamento, estratégia essa defendida em vários estudos que mostram sua eficácia.

O isolamento social afetou várias áreas da saúde, inclusive a fisioterapia, transformando-se em uma barreira aos profissionais que estão em contato próximo ao paciente, a exemplo temos aqueles que se recuperam de fraturas, pacientes oncológicos, os que apresentam síndrome de Down, entre outros.

Com o objetivo de facilitar a prestação de serviço de fisioterapia em tempos de pandemia e o acesso a informações nos cuidados com a saúde o Conselho Federal de Fisioterapia e Terapia Manual de acordo com diretrizes de entidades internacionais, permitiu o atendimento por meio de tele consultas e tele monitoramento. (Coffito, 2020)

Estudos como o de Agostini et al. (2015) e Dávila Castrodad et al. (2019) encontraram resultados promissores ao implantar a telerreabilitação em pacientes após artroplastia total como ombro, joelho e quadril além de intervenção de membros superiores como fratura de úmero proximal, cirurgia de túnel do carpo e ruptura do manguito rotador.

Em seu estudo, Turolla et al. (2020) abrange vários desafios presentes na telerreablitação, dentre eles destaca a importância de uma boa comunicação verbal como, instruções claras e expressões de apoio e na comunicação não-verbal que abrange, aceno da cabeça, postura corporal e contato visual.

Outra barreira destacada pelo autor é a falta de equipamentos a serem utilizados no tratamento, para resolver tal limitação o autor aconselha que durante o planejamento inicial do programa de reabilitação, o fisioterapeuta estabeleça quais ferramentas serão utilizadas para a realização de exercícios terapêuticos, de forma que possam, por exemplo, ser alugadas e entregues na casa do paciente. 
Minghelli (2020) elencou em seu estudo as principais medidas utilizadas pelos fisioterapeutas para acompanhar os seus pacientes à distância. Dentre elas a mais encontrada foi a prescrição do tratamento por escrito, em seguida observou-se a realização de vídeos explicativos e por último o tratamento por videoconferência de forma simultânea.

Com o avanço da tecnologia, hoje por meio de celular, computador ou tablete é possível aplicar, em alguns casos, a tele consulta, assim como o tele monitoramento. Lee (2020) indica a teleconsulta em atividades educativas, orientações de atividades para autocuidado e em casos avaliados previamente, a continuação da cinesioterapia.

Eccleston et al. (2020) vai mais longe e afirma que embora existam limitações impostas pela tele consulta ao substituir o exame físico, o exame virtual é capaz de observar aparências, movimentos, além de, sob orientação, ser possível realizar autoexame.

De Moura Carvalho et al. (2020) em seu estudo com pacientes oncológicos aponta a importância da tele consulta no que tange evitar a descontinuação do acompanhamento, pois consegue-se por meio da internet reduzir o trânsito para o hospital e afins, assim como o risco de contaminação e sem dúvidas favorecendo a funcionalidade do paciente.

Existem três formas de atendimento fisioterapêutico, a primeira é a forma presencial onde o paciente desloca-se até a clínica, hospital ou ambulatório, a segunda é quando por algum motivo o paciente não pode deslocar-se então o profissional vai até sua casa e por último, devido ao cenário pandêmico, ocorre o atendimento por meio de plataformas digitais. Este último ao que tudo indica é transitório, mas para Soares e Fiorindo (2020) esse sistema deve ser visto como modelo efetivo a ser utilizado na rotina do fisioterapeuta, pois torna-se mais um meio colocado à disposição para garantir melhor qualidade de vida ao paciente.

\subsection{Barreiras e dificuldades para um uso ampliado do teleatendimento no manuseio da pandemia da covid-19}

Turolla e colaboradores (2020), afirmam que as estratégias de isolamento e distanciamento social, apesar de ser medidas necessárias para reduzir os danos da pandemia da COVID-19 e evitar o colapso dos sistemas de saúde, essa mudança de paradigma constitui uma barreira para os profissionais da saúde, em especial aos fisioterapeutas traumato-ortopédicos, que necessitam do contato próximo com os pacientes que precisam de cuidados em dores crônicas musculoesqueléticas. Em contrapartida, eles destacam que este período de pandemia é um momento propício para se explorar o potencial da telessaúde para pacientes com distúrbios musculoesqueléticos. No entanto, afirmam que esse novo campo de atuação profissional, traz alguns desafios que ainda não foram debatidos a fundo. Um exemplo são os elementos específicos de comunicação verbal e não verbal (aceno de cabeça afirmativo, contato visual, contato com outros pacientes) de forma adaptada à individualidade do paciente, que é possível ser visto nos atendimentos presenciais. Outra barreira encontrada foi em relação aos equipamentos, a falta de instrumentos de reabilitação (como faixas elásticas, pesos, bolas) na casa do paciente que podem limitar prestação de cuidados durante os atendimentos remotos.

De acordo com Mauri e colaboradores (2020), a maioria dos serviços de fisioterapia na Itália foram suspensos, e os atendimentos remotos não foram fornecidos, possivelmente por causa da dificuldade de vínculos vivenciados por pacientes com deficiência e seus cuidadores para auxiliar na realização das sessões de fisioterapia de forma não presencial. Com isso, eles afirmam que o uso da telessaúde pode ser útil em um futuro próximo após a fase aguda da pandemia, onde já poder haver uma padronização dos atendimentos remotos em fisioterapia.

Márquez (2020) reitera em seu estudo que o uso ampliado da telessaúde no Brasil é uma estratégia sólida para superar obstáculos como a má distribuição dos serviços de saúde e a presença desigual de profissionais no território, obstáculos esses que geram longas filas de espera por atendimento, principalmente na saúde pública. Por outro lado, a legislação brasileira pode ser um empecilho ao restringir as teleconsultas e a infraestrutura tecnológica do país é escassa, o que configura em uma limitação de acesso à internet de qualidade em localidades remotas. Então o acesso restrito à tecnologia como smartphone, 
tablet, computador e plataformas adequadas pode resultar em barreiras para o uso da telessaúde, bem como grupos populacionais como idosos ou pessoas com baixa escolaridade que não possuem conhecimento para a correta utilização da tecnologia.

Minghelli e colaboradores (2020), corrobora com o estudo de Márquez, no sentido de que a dificuldade de acesso à tecnologia traz desafios importantes no uso ampliado da telessaúde. Corroborando também com o estudo de Turolla, Minghelli afirma que algumas adaptações nas características dos atendimentos remotos de fisioterapia tiveram que ser feitas, pois a maioria dos pacientes não apresentam os equipamentos ou materiais necessários para a sua prática.

\section{Considerações Finais}

A telessaúde tem sido uma importante alternativa para os profissionais da saúde garantirem o acompanhamento de seus pacientes em tempo real neste período de pandemia da COVID-19, tornando-se uma possibilidade para superar os danos à saúde causados pelo isolamento social. Todavia, esta ferramenta detém aspectos negativos como a limitação de acesso à tecnologia e Internet de qualidade, falta de plataformas padronizadas e seguras, comunicação limitada, falta de instrumentos de reabilitação na casa do paciente e dificuldades na realização dos exercícios durante os atendimentos fisioterapêuticos, principalmente em pacientes com alguma limitação funcional. Outro desafio importante está atrelado aos aspectos éticos que devem ser levados em consideração, onde um dos pontos principais é a proteção de dados do paciente. Sendo assim, os atendimentos fisioterapêuticos de forma remota, se depara com desafios significativos que devem ser estudados a fundo e o uso da telessaúde pode ser mais proficiente em um futuro bem próximo. O presente trabalho teve limitações devido a carência de estudos na área. Portanto, sugerimos um maior aprofundamento em pesquisas sobre esta modalidade de atendimento principalmente em países subdesenvolvidos, nos quais o acesso a Internet de qualidade é limitado.

\section{Referências}

Agostini, M., Moja, L., Banzi, R., Pistotti, V., Tonin, P., Venneri, A., \& Turolla, A. (2015). Telerehabilitation and recovery of motor function: a systematic review and meta-analysis. Journal of telemedicine and telecare, 21(4), 202-213. https://doi.org/10.1177\%2F1357633X15572201

Brasil. (2020). Declara Emergência em Saúde Pública de importância Nacional (ESPIN) em decorrência da Infecção Humana pelo novo Coronavírus (2019nCoV). Portaria $n^{\circ}$ 188, de 3 de fevereiro de 2020,1-1. http://www.planalto.gov.br/ccivil_03/portaria/prt188-20-ms.htm

Conselho Federal De Fisioterapia E Terapia Ocupacional (BR). Resolução n ${ }^{\circ}$ 516, de 20 de março de 2020. aplicável sobre a suspensão temporária do Artigo 15, inciso II e Artigo 39 da Resolução COFFITO nº 424/2013 e Artigo 15, inciso II e Artigo 39 da Resolução COFFITO no $425 / 2013$ e outras providências durante o enfrentamento da crise provocada pela Pandemia do COVID-19 [Internet]. Diário Oficial da União, Brasília, DF; 2020 mar 23. Seção I, p. 184. [acesso 2020 outubro 29]. Disponível em: http://www.crefito2.gov.br/comunicados/ comunicados / coffito-publicaresolucao-n-516-2020- permitindo-at3144.html.

Castrodad, I. M. D., Recai, T. M., Abraham, M. M., Etcheson, J. I., Mohamed, N. S., Edalatpour, A., \& Delanois, R. E. (2019). Rehabilitation protocols following total knee arthroplasty: a review of study designs and outcome measures. Annals of translational medicine, 7(Suppl 7). 10.21037 / atm.2019.08.15

de Moura Carvalho, R. B., Ferreira, K. R., \& Modesto, F. C. (2020). A fisioterapia digital em oncoginecologia durante a pandemia de Covid-19. Revista Brasileira de Cancerologia, 66(TemaAtual). https://doi.org/10.32635/2176-9745.RBC.2020v66nTemaAtual.1082

Eccleston, C., Blyth, F. M., Dear, B. F., Fisher, E. A., Keefe, F. J., Lynch, M. E., \& de C Williams, A. C. (2020). Managing patients with chronic pain during the COVID-19 outbreak: considerations for the rapid introduction of remotely supported (eHealth) pain management services. Pain, 161(5), 889. https://dx.doi.org/10.1097\%2Fj.pain.0000000000001885

Lee, A. C. (2020). COVID-19 and the advancement of digital physical therapist practice and telehealth. Physical therapy, 100(7), 1054-1057. https://doi.org/10.1093/ptj/pzaa079

Lloyd-Sherlock, P., Ebrahim, S., Geffen, L., \& McKee, M. (2020). Bearing the brunt of covid-19: older people in low and middle income countries. https://doi.org/10.1136/bmj.m1052

Márquez, J. R. (2020). Teleconsultation in a pandemic due to Coronavirus: challenges for telemedicine in the post-COVID-19 era. Revista Colombiana de Gastroenterologia, 35, 5-16. https://doi.org/10.22516/25007440.543

Mauri, E., Abati, E., Musumeci, O., Rodolico, C., D’Angelo, M. G., Mirabella, M., ... \& of Myology, I. A. (2020). Estimating the impact of COVID-19 pandemic on services provided by Italian Neuromuscular Centers: an Italian Association of Myology survey of the acute phase. Acta Myologica, $39(2), 57$. https://dx.doi.org/10.36185\%2F2532-1900-008 
Research, Society and Development, v. 10, n. 12, e119101220130, 2021

(CC BY 4.0) | ISSN 2525-3409 | DOI: http://dx.doi.org/10.33448/rsd-v10i12.20130

Medeiros, E. A. S. (2020). A luta dos profissionais de saúde no enfrentamento da COVID-19. < https://www.scielo.br/j/ape/a/Nc8yzcvt rvXbWBgBGskm36S/?lang=pt\&format=pdf $>$.

Mendes, K. D. S.; Silveira, R. C. de C. P.; Galvão, C. M. Revisão integrativa: método de pesquisa para a incorporação de evidências na saúde e na enfermagem. Texto \& contexto-enfermagem, 17, 758-764, 2008. https://doi.org/10.1590/S0104-07072008000400018

Minghelli, B., Soares, A., Guerreiro, A., Ribeiro, A., Cabrita, C., Vitoria, C., \& Antunes, R. (2020). Physiotherapy services in the face of a pandemic. Revista da Associação Médica Brasileira, 66, 491-497. https://doi.org/10.1590/1806-9282.66.4.491

Mizumoto, K., Kagaya, K., Zarebski, A., \& Chowell, G. (2020). Estimating the asymptomatic proportion of coronavirus disease 2019 (COVID-19) cases on board the Diamond Princess cruise ship, Yokohama, Japan, 2020. Eurosurveillance, 25(10), 2000180. https://www.eurosurveillance.org/content/10.2807/1560-7917.ES.2020.25.10.2000180/

Pegorari, M. S., Ohara, D. G., Matos, A. P., \& Pinto, A. C. P. N. (2020). Covid-19: perspectives and initiatives in older adults health context in Brazil. Ciencia \& saude coletiva, 25 , 3459-3464. https://doi.org/10.1590/1413-81232020259.21622020

Soares, G. P., Fiorindo, V. F. (2020). Direito e fisioterapia: reflexões acerca dos atendimentos fisioterápicos não presenciais à luz do direito à saúde do paciente em tempos pandêmicos. ETIC-Encontro de Iniciação Científica-issn 21-76-8498, 16(16). http://intertemas.toledoprudente.edu.br/index.php/ETI C/article/view/8750

Surveillances, V. (2020). The epidemiological characteristics of an outbreak of 2019 novel coronavirus diseases (COVID-19) - China, 2020. China CDC weekly, 2(8), 113-122. http://www.het-vl.com.tw/system/files/1090327.pdf

Shahid, Z., Kalayanamitra, R., McClafferty, B., Kepko, D., Ramgobin, D., Patel, R., \& Jain, R. (2020). COVID-19 and older adults: what we know. Journal of the American Geriatrics Society, 68(5), 926-929. https://doi.org/10.1111/jgs.16472

Turolla, A., Rossettini, G., Viceconti, A., Palese, A., \& Geri, T. (2020). Musculoskeletal physical therapy during the COVID-19 pandemic: is telerehabilitation the answer? Physical therapy, 100(8), 1260-1264. https://doi.org/10.1093/ptj/pzaa093

World Health Organization. (2020). Coronavirus disease 2019 (COVID-19): situation report, 73. https://www.paho.org/pt/covid19

Zhang, W. (2020). Manual de prevenção e controle da Covid-19 segundo o Doutor Wenhong Zhang. PoloBooks. https://www.forumchinaplp.org.mo/wpcontent/uploads/2020/10/Manual-de-Preven\%C3\%A7\%C3\%A3o_PT.pdf 